\title{
Estilos de disciplina y trastornos comórbidos de adolescentes con trastorno por déficit de atención/hiperactividad. Un estudio longitudinal.
}

ARTICLE in REVISTA DE NEUROLOGIA · JANUARY 2014

Impact Factor: 0.93

4 AUTHORS, INCLUDING:

Raul Tarraga

University of Valencia

27 PUBLICATIONS 31 CITATIONS

SEE PROFILE
Ana Miranda

University of Valencia

140 PUBLICATIONS $\quad 2,665$ CITATIONS

SEE PROFILE 


\title{
Estilos de disciplina y trastornos comórbidos de adolescentes con trastorno por déficit de atención/hiperactividad. Un estudio longitudinal
}

\author{
Carla Colomer-Diago, Carmen Berenguer-Forner, Raúl Tárraga-Mínguez, Ana Miranda-Casas
}

Introducción. Los problemas en el funcionamiento cognitivo, social y educativo de los niños con trastorno por déficit de atención/hiperactividad (TDAH) continúan estando presentes en la adolescencia y edad adulta. A pesar de que la bibliografía evidencia una relación significativa entre la utilización de métodos de disciplina disfuncional y la gravedad en la evolución del TDAH, las investigaciones de seguimiento han sido escasas.

Objetivos. Analizar el poder del estilo de disciplina y de la sintomatología de TDAH evaluados en la niñez (tiempo 1) para predecir el oposicionismo y los problemas cognitivos en la temprana adolescencia (tiempo 2), y estudiar, en función del uso de un estilo de disciplina disfuncional, la evolución del oposicionismo y de los problemas cognitivos.

Pacientes y métodos. Se evaluó a 45 niños con TDAH subtipo combinado en dos momentos temporales: tiempo 1 (6-13 años) y tiempo 2 (8-16 años).

Resultados. La gravedad del TDAH y el estilo de crianza disfuncional predijeron tanto el oposicionismo como los problemas cognitivos estimados en el tiempo 2. Se encontró un aumento del oposicionismo entre el tiempo 1 y el tiempo 2 en el grupo de disciplina disfuncional, mientras que el efecto contrario se observó en el grupo de disciplina funcional (efecto de interacción tiempo $\times$ estilo de disciplina).

Conclusiones. El empleo de un estilo de disciplina disfuncional por parte de los padres en la niñez predice problemas cognitivos y de conducta en la adolescencia. Los hallazgos tienen implicaciones en la planificación de las intervenciones.

Palabras clave. Estilos de disciplina. Estudio longitudinal. Problemas cognitivos. Problemas de comportamiento. TDAH.

\section{Introducción}

La presentación de forma aislada de las manifestaciones nucleares del trastorno por déficit de atención/hiperactividad (TDAH) -esto es, desatención, hiperactividad e impulsividad- resulta poco frecuente. Según el estudio de Kadesjo y Gillberg [1], un $87 \%$ de niños que cumplían criterios de TDAH tenían, al menos, un diagnóstico comórbido, destacando por su frecuencia el trastorno negativista/ desafiante y el trastorno del desarrollo de la coordinación. En otro trabajo a gran escala en el que participaron más de 5.000 niños con TDAH, Larson et al [2] encontraron que un $67 \%$ tenían al menos otro trastorno mental o del neurodesarrollo, en comparación con un $11 \%$ del resto de niños, destacando la elevada asociación con el trastorno de conducta (27\% frente a $2 \%$ ) y con las dificultades de aprendizaje (46\% frente a $5 \%)$.

Los problemas en el funcionamiento educativo, social y de salud mental de los niños con TDAH continúan estando presentes, e incluso se acentúan, en la adolescencia y en la edad adulta. Así lo atestigua un estudio de Huh et al [3] en el que participaron 232 sujetos divididos en dos grupos de edad: niños (5-12 años) y adolescentes (12-17 años); se hallaron tasas de comorbilidad en el grupo de adolescentes (70\%) significativamente más altas que las del grupo de niños con TDAH (48\%), sobre todo en los trastornos de conducta. La asociación entre TDAH y trastornos de conducta es especialmente preocupante porque explica en gran medida los resultados negativos relacionados con la comisión temprana de actos delictivos (asalto, incendio, violación...) y con el abuso de sustancias $[4,5]$. Pero, en cualquier caso, los estudios de seguimiento indican que los sujetos con TDAH y trastornos comórbidos tienen una evolución peor y experimentan más dificultades sociales, emocionales y psicológicas [6].

La comprensión de los factores que están implicados en la evolución del TDAH es hoy por hoy un reto, debido a las complejas y múltiples interacciones de factores genéticos y ambientales que se producen a lo largo del desarrollo. El gen receptor de la
Departamento de Psicología Evolutiva y de la Educación (C. Colomer-Diago, C. BerenquerForner, A. Miranda-Casas); Departamento de Didáctica y Organización Escolar (R. TárragaMínguez). Universitat de València. Valencia, España.

Correspondencia: Dra. Ana Miranda Casas. Departamento de Psicología Evolutiva y de la Educación. Universitat de València. Avda. Blasco Ibáñez, 21. E-46010 Valencia.

E-mail: ana.miranda@uv.es

Financiación: Trabajo financiado por el MICINN (ref.: BES-2010-033019).

Declaración de intereses: Los autores declaran la inexistencia de conflictos de interés en relación con este artículo.

Aceptado tras revisión externa: 10.01.14.

Cómo citar este artículo: Colomer-Diago C, BerenguerForner C, Tárraga-Mínguez R, Miranda-Casas A. Estilos de disciplina y trastornos comórbidos de adolescentes con trastorno por déficit de atención/hiperactividad. Un estudio longitudinal. Rev Neurol 2014; 58 (Supl 1): S31-6.

(c) 2014 Revista de Neurología 
dopamina (DRD4) puede ser un factor de riesgo de TDAH, aumentando la sensibilidad del niño ante contingencias ambientales, como un estilo de crianza disfuncional. Se ha comprobado que la expresión conductual de niños con alelo 7 repetido cambia en función del estilo de crianza de los padres, de manera que los padres con un estilo de crianza óptimo tienen hijos con puntuaciones inferiores en el rasgo de 'búsqueda de sensaciones' -que está relacionado con el TDAH- a las de hijos de padres con un estilo de crianza menos cualificado [7]. Por el contrario, los niños con repetición del alelo 7 despliegan, en el contexto de una paternidad negativa, una capacidad menor de suprimir una respuesta dominante para ejecutar una respuesta subdominante que los niños sin este alelo [8].

En este sentido resulta muy clarificadora la revisión de Pennington [9], basada en un número cuantioso de estudios genéticos. Pennington concluyó que los niños con sólo TDAH y los niños que tenían trastornos de conducta asociados presentaban perfiles genéticos muy similares, lo cual estaba sugiriendo el importante papel de los factores ambientales en su evolución: 'con una paternidad óptima, un niño con ese perfil genético desarrollará sólo un TDAH; con una paternidad nefasta, el mismo niño también desarrollará un trastorno de conducta'.

La bibliografía respalda la relación significativa entre utilización de métodos de disciplina disfuncional - permisivos, impositivos y sobrerreactivos-y gravedad en la evolución de los síntomas del TDAH [10]. Se produce además una cierta especificidad en dicha relación, en función de los diferentes puntos del desarrollo. Como muestra el estudio de seguimiento de 12 meses de Hawes et al [11], el empleo de prácticas de disciplina efectivas mejora la capacidad de autorregulación en edades tempranas, mientras que, por el contrario, los patrones de disciplina más inconsistentes contribuyen a agravar la sintomatología nuclear del TDAH en la niñez media.

El uso de métodos de disciplina negativos o poco efectivos por parte de los padres de niños con TDAH también se asocia a altos niveles de problemas de conducta, oposicionismo desafiante y rechazo de los compañeros. Más específicamente, aunque las madres de niños con TDAH no sean más permisivas o inconsistentes que las madres de niños sin TDAH, suelen aplicar estrategias de disciplina emocionales, mostrándose más autoritarias y reaccionando con mayor irritabilidad y frustración, sobre todo en presencia de problemas de oposicionismo [12,13]. Kaiser et al [14] se propusieron profundizar en la posibilidad de la gravedad del TDAH y del estilo de paternidad (positiva/negativa) para predecir las habilidades sociales y el comportamiento agresivo del niño. Para ello pusieron a prueba tres posibles modelos: de efectos principales, de mediación y de moderación en una muestra de 143 niños de 5-11 años con y sin TDAH. Los análisis aportaron efectos principales significativos de la gravedad del TDAH y del estilo de disciplina en las variables del funcionamiento social del niño. También hubo alguna evidencia de un efecto de mediación del estilo de paternidad negativa en la relación entre gravedad del TDAH y agresividad y habilidad social del niño, mientras que no se observó un efecto de moderación.

Los comentarios anteriores ilustran el progresivo reconocimiento de la influencia del estilo de disciplina en la expresión del TDAH y de los problemas asociados. Paradójicamente, la investigación longitudinal que ha analizado la posible trayectoria evolutiva ha sido muy escasa. En particular, profundizar en el conocimiento de los procesos de socialización familiar que influyen en el curso del TDAH impulsaría a diseñar una actuación más efectiva ante el trastorno.

El interés fundamental del presente estudio ha sido examinar la influencia de los estilos de disciplina y de los síntomas de TDAH en el desarrollo del oposicionismo y de los problemas cognitivos entre la niñez media (tiempo 1) y la adolescencia temprana (tiempo 2). Un primer objetivo consistió en analizar el poder del estilo de disciplina y la sintomatología del TDAH evaluados en la niñez (tiempo 1) para predecir el oposicionismo y los problemas cognitivos en la temprana adolescencia (tiempo 2). El segundo objetivo fue estudiar, en función del uso de un estilo de disciplina disfuncional y de la presencia de sintomatología de TDAH grave, la evolución del oposicionismo y de los problemas cognitivos entre el tiempo 1 y el tiempo 2. En base a la evidencia aportada por estudios con diseños generalmente transaccionales se hipotetizó que los estilos de disciplina y los síntomas de TDAH predecirían tres años después los problemas cognitivos y de conducta asociados. Además, los niños cuyos padres usaran un estilo de disciplina disfuncional en la niñez mostrarían peores resultados en oposicionismo y en problemas cognitivos en la adolescencia.

\section{Pacientes y métodos}

\section{Participantes}

En este estudio participaron 45 niños con un diagnóstico de TDAH subtipo combinado, que forma- 
ron parte de la muestra del proyecto IMAGE (The International Multicentre ADHD Genetics Project). Los participantes presentaban, de acuerdo con la percepción de los padres, seis o más de los síntomas de desatención y de hiperactividad/impulsividad del Manual diagnóstico y estadístico de los trastornos mentales, cuarta edición, texto revisado (DSM-IV-TR), con una sintomatología superior a seis meses y aparición de las alteraciones antes de los 7 años. En la selección de la muestra se incluyeron niños con un cociente intelectual $(\mathrm{CI})>80$, que no presentaran problemas psiquiátricos, neurológicos, déficits sensoriales o motores.

Los participantes del estudio fueron evaluados en una primera fase (abril de 2003 a julio de 2004) y tres años después en una fase de seguimiento. En la fase 1 se realizó el diagnóstico clínico de TDAH subtipo combinado de 45 niños con edades de 6-12 años (media: 8,22 $\pm 1,76$ años), un CI dentro de los rangos de la normalidad (CI medio: 106,99 $\pm 16,60$ ) y predominio de varones $(93,3 \%)$. El rango de edad de los sujetos en la fase de seguimiento, tres años después, fue de 8-15 años (media: 10,46 $\pm 1,98$ años).

Considerando la percepción de los padres, un alto porcentaje de los participantes presentaban puntuaciones $T \geq 63$ en la escala de Conners para padres en oposicionismo (64,4\% en el tiempo 1 y $44,4 \%$ en el tiempo 2) y en problemas cognitivos y de rendimiento (93,3\% en el tiempo 1 y $77,8 \%$ en el tiempo 2).

En cuanto a características familiares, un porcentaje minoritario de la muestra se trataba de familias monoparentales $(15,6 \%$ frente a $84,4 \%$ con dos progenitores), siendo la madre la principal figura de crianza $(66,7 \%)$. Un $60 \%$ de los padres consideraban que no tenían conocimientos suficientes sobre el TDAH.

\section{Instrumentos}

\section{Escala de estilos de disciplina [15]}

Se trata de una escala de evaluación diseñada para medir las prácticas disfuncionales de disciplina. Consta de 30 ítems, en los que los padres valoran la probabilidad de utilizar una estrategia determinada de disciplina ante comportamientos inadecuados del niño, debiendo situar su respuesta entre dos opciones opuestas en una escala de siete puntos tipo Likert: la puntuación de 1 indica una alta probabilidad de utilizar una estrategia de disciplina eficaz, y la puntuación de 7 , una alta probabilidad de usar una estrategia de disciplina disfuncional.

Los ítems conforman estas tres subescalas:
- Disciplina permisiva. Escasa exigencia y control sobre los hijos; se cede a las exigencias del hijo o se refuerza el comportamiento inadecuado del niño: 'si mi hijo se enfada cuando le niego algo..,' 'doy marcha atrás y cedo' (7) frente a 'mantengo la negativa' (1).

- Disciplina autoritaria/emocional. Se reacciona con excesiva frustración, irritabilidad y enfado: 'cuando mi hijo se porta mal...' 'le hablo muy alto o le grito' (7) frente a 'le hablo con calma, serenamente' (1).

- Disciplina con verborrea. Abundancia de amenazas y recriminaciones a pesar de su ineficacia: 'cuando surge un problema con mi hijo..,' 'le hago muchas advertencias antes de actuar' (7) frente a "le advierto de lo que va a ocurrir sólo una vez' (1).

Las puntuaciones de la escala correlacionan significativamente con medidas observacionales de disciplina; la escala tiene una buena fiabilidad test-retest y su estructura factorial es coherente con la investigación desarrollada sobre estilos de paternidad [16].

\section{Escala de Conners para padres [17]}

Contiene 80 ítems que se valoran mediante una escala tipo Likert de cuatro puntos. De las 14 subescalas del cuestionario se seleccionaron para esta investigación las subescalas de oposicionismo (p. ej., es desafiante), de problemas cognitivos/desatención (p. ej., olvida cosas que había aprendido) y la escala del DSM-IV total que refleja fielmente los criterios del DSM-IV sobre el TDAH: inatención (p. ej., parece que no escucha lo que se le dice) e hiperactividad/impulsividad (p. ej., tiene dificultades para esperar su turno). Las escalas de Conners se encuentran entre los instrumentos de medida para el TDAH con mejor estandarización, validez y fiabilidad [18].

\section{Procedimiento}

En el tiempo 1 o primera fase se realizó el diagnóstico de TDAH subtipo combinado y se administró la escala de Conners para padres con el fin de evaluar la sintomatología del TDAH y los principales problemas asociados. También se administró la escala de estilos de disciplina de Arnold et al [15] para identificar las prácticas de disciplina en los padres. Tres años después, en la fase 2 de seguimiento, se recogió de nuevo información sobre síntomas de TDAH y problemas conductuales y cognitivos. Ambas evaluaciones se realizaron con el consentimiento informado de las familias. 
Tabla. Resultados de los análisis de regresión múltiple de los síntomas del TDAH y del estilo de disciplina sobre el oposicionismo y problemas cognitivos en el tiempo 2.

\begin{tabular}{|c|c|c|c|c|c|}
\hline & & $R$ & $R^{2}$ & $\begin{array}{c}\text { Sintomatología } \\
\text { de TDAH } \\
\text { (estadístico } t \text { ) }\end{array}$ & $\begin{array}{c}\text { Estilo de } \\
\text { disciplina } \\
\text { (estadístico } t \text { ) }\end{array}$ \\
\hline \multirow{2}{*}{$\begin{array}{l}\text { Estilo de } \\
\text { disciplina } \\
\text { disfuncional }\end{array}$} & Oposicionismo & 0,546 & 0,298 & $3,21^{b}$ & $2,36^{a}$ \\
\hline & Problemas cognitivos & 0,552 & 0,304 & $3,16^{b}$ & $2,52^{a}$ \\
\hline \multirow{2}{*}{$\begin{array}{l}\text { Estilo } \\
\text { autoritario }\end{array}$} & Oposicionismo & 0,540 & 0,291 & $3,52^{b}$ & $2,26^{a}$ \\
\hline & Problemas cognitivos & 0,562 & 0,316 & $3,54^{b}$ & $2,68^{b}$ \\
\hline \multirow{2}{*}{$\begin{array}{l}\text { Estilo } \\
\text { permisivo }\end{array}$} & Oposicionismo & 0,557 & 0,310 & $3,13^{b}$ & $2,53^{a}$ \\
\hline & Problemas cognitivos & 0,505 & 0,255 & $3,06^{b}$ & 1,77 \\
\hline \multirow{2}{*}{$\begin{array}{l}\text { Disciplina } \\
\text { con verborrea }\end{array}$} & Oposicionismo & 0,456 & 0,208 & $3,25^{b}$ & 0,42 \\
\hline & Problemas cognitivos & 0,454 & 0,206 & $3,18^{b}$ & 0,61 \\
\hline
\end{tabular}

${ }^{\mathrm{a}} p<0,05 ;{ }^{\mathrm{b}} p<0,01$. nismo y el $30,4 \%$ de la varianza total de problemas cognitivos (Tabla).

El estilo de disciplina autoritario fue un predictor significativo, junto con la gravedad de TDAH, tanto del oposicionismo (sintomatología de TDAH: $t=3,52, p=0,001$; estilos de disciplina: $t=2,26, p=$ $0,029)$ como de los problemas cognitivos (sintomatología de TDAH: $t=3,54, p=0,001$; estilos de disciplina: $t=2,68, p=0,010$ ), explicando el $29,1 \%$ y el $31,6 \%$ de sus varianzas totales, respectivamente. Por su parte, el estilo de disciplina permisivo $(t=2,53$, $p=0,015)$, junto con la gravedad del TDAH $(t=3,13$, $p=0,003)$, predijo el $31 \%$ de la varianza total de oposicionismo, sin encontrar relaciones estadísticamente significativas con la variable de problemas cognitivos. La disciplina con verborrea no predijo ninguno de los problemas comórbidos analizados.

La figura muestra los resultados de los ANOVA mixtos. El ANOVA para evaluar el efecto del estilo de disciplina (funcional frente a disfuncional) sobre la evolución del oposicionismo no encontró diferencias estadísticamente significativas entre el estilo de disciplina $\left(F_{1,43}=0,01 ; p=0,962 ; \eta_{p}^{2}=0,01\right)$ ni entre el tiempo $\left(F_{1,43}=0,651 ; p=0,424 ; \eta_{p}^{2}=\right.$ $0,02)$. Sin embargo, el efecto de interacción sí resultó estadísticamente significativo $\left(F_{1,43}=15,58 ; p<\right.$ 0,$001 ; \eta_{p}^{2}=0,27$ ) (Figura, a).

El segundo ANOVA evaluó el efecto de la gravedad del TDAH (grave frente a no grave) sobre la evolución del oposicionismo. Los resultados no reflejaron diferencias estadísticamente significativas entre el tiempo $\left(F_{1,43}=0,37 ; p=0,545 ; \eta_{p}^{2}=0,01\right)$ ni en la interacción $\left(F_{1,43}=2,37 ; p=0,131 ; \eta_{\mathrm{p}}^{2}=\right.$ $0,05)$. La gravedad del TDAH sí mostró diferencias significativas $\left(F_{1,43}=5,27 ; p=0,027 ; \eta_{\mathrm{p}}^{2}=0,11\right)(\mathrm{Fi}$ gura, b).

Los resultados del efecto del estilo de disciplina sobre la evolución de los problemas cognitivos no mostraron diferencias estadísticamente significativas entre el tiempo $\left(F_{1,43}=1,35 ; p=0,251 ; \eta_{\mathrm{p}}^{2}=\right.$ $0,03)$. El efecto del estilo de disciplina se quedó cerca de la significación $\left(F_{1,43}=3,41 ; p=0,072 ; \eta_{p}^{2}=\right.$ $0,07)$, mostrando una tendencia del grupo con un estilo de disciplina disfuncional a presentar más problemas cognitivos que el grupo con una disciplina funcional. A pesar de que no se encontró efecto de interacción de tiempo $\times$ estilo de disciplina $\left(F_{1,43}=\right.$ 2,$\left.50 ; p=0,121 ; \eta_{\mathrm{p}}^{2}=0,05\right)$, se constató una tendencia a la disminución de problemas cognitivos del tiempo 1 al tiempo 2 en el grupo con una disciplina funcional, mientras que se observó un ligero aumento de las puntuaciones de problemas cognitivos en el grupo con un estilo de disciplina disfuncional (Figura, c). 
Finalmente, los análisis relacionados con el efecto de la gravedad del TDAH sobre la evolución de los problemas cognitivos mostraron únicamente un efecto de grupo (grave frente a no grave) $\left(F_{1,43}=8,21\right.$; $\left.p=0,006 ; \eta_{\mathrm{p}}^{2}=0,16\right)$. No se encontraron efectos de tiempo $\left(F_{1,43}=1,27 ; p=0,265 ; \eta_{\mathrm{p}}^{2}=0,03\right)$ ni de interacción $\left(F_{1,43}=0,19 ; p=0,661 ; \eta_{\mathrm{p}}^{2}=0,01\right)$ (Figura, $\mathrm{d}$ ).

\section{Discusión}

El diseño longitudinal de este estudio ha permitido profundizar el papel que desempeñan el estilo de disciplina de la familia y la sintomatología de TDAH en los problemas de oposicionismo y cognitivos en la primera adolescencia. En cuanto a los estilos de disciplina específicos, el autoritarismo, junto con la sintomatología de TDAH, mostró poder para predecir las conductas oposicionistas y los problemas cognitivos. También la permisividad y los síntomas de TDAH predijeron un porcentaje significativo de la varianza total de oposicionismo, aunque las relaciones con los problemas cognitivos no fueron estadísticamente significativas. Por el contrario, la disciplina basada en la verborrea no mostró relación con ninguno de los problemas comórbidos que se analizaron.

Por otra parte, el uso de un estilo de disciplina funcional produjo un descenso de conductas de oposicionismo y de los problemas cognitivos desde la niñez a la primera adolescencia, mientras que con la aplicación de un estilo de disciplina disfuncional se observó el efecto contrario. Por su parte, la gravedad del TDAH se relacionó con mayor sintomatología de oposicionismo y de problemas cognitivos tanto en la niñez como en la primera adolescencia.

En resumen, coincidiendo con trabajos previos realizados con diseños transaccionales [14,19,20], nuestros hallazgos muestran que el estilo de disciplina autoritario $y$, en menor medida, el estilo permisivo de los padres, junto con la gravedad del TDAH, están implicados en el desarrollo del oposicionismo y de los problemas cognitivos en la adolescencia. Por consiguiente, la opción de tratamiento para controlar los problemas conductuales y cognitivos asociados al TDAH consistirá en una intervención multimodal que aborde la reducción de los síntomas y mejore los estilos de disciplina de la familia.

El diseño longitudinal para aclarar posibles vínculos causales es el principal mérito de esta investigación. Pero también está afectada por una serie de limitaciones que futuros trabajos habrán de subsa-
Figura. Efectos del estilo de crianza y de la gravedad de la sintomatología del TDAH sobre la evolución del oposicionismo $(a, b)$ y de los problemas cognitivos $(c, d)$ de adolescentes con TDAH.

\section{a}

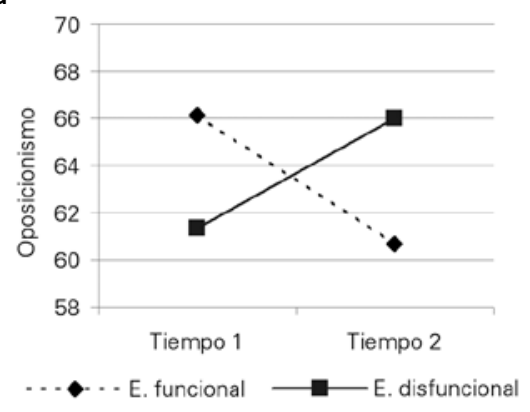

b

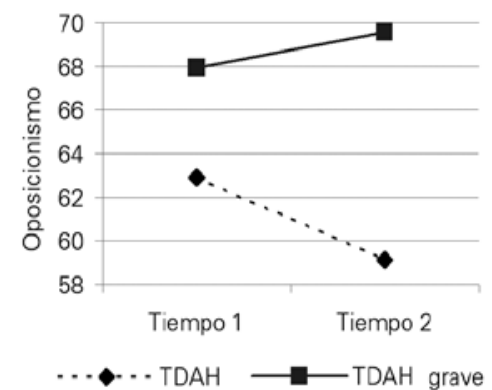

d
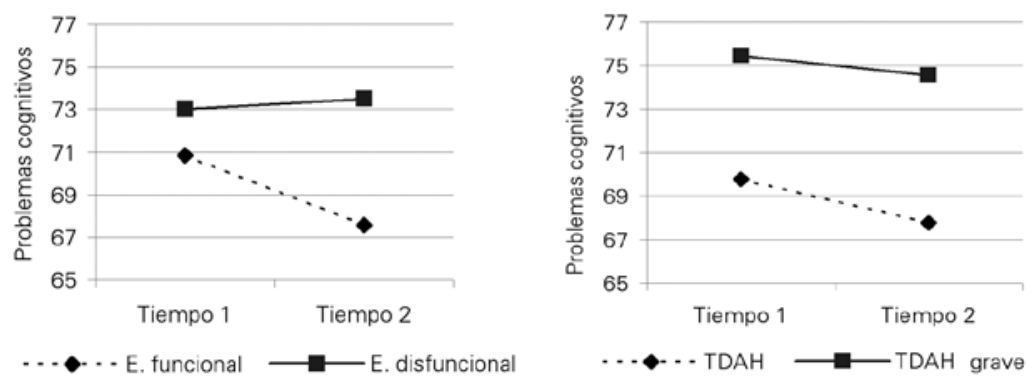

nar, entre otras, el reducido número de participantes, la falta de un grupo de comparación sin TDAH o el hecho de que la información sobre los estilos de disciplina se recogiera a partir de cuestionarios cumplimentados sobre todo por las madres y no mediante la observación de las interacciones padres/ hijo.

\section{Bibliografía}

1. Kadesjo B, Gillberg C. The comorbidity of ADHD in the general population of Swedish school-children age. J Child Psychol Psychiatry 2001; 42: 487-92.

2. Larson K, Russ S, Kahn, RS, Halfon N. Pattern of comorbidity, functioning, and service use for US children with ADHD. Pediatrics 2011; 127: 1-9.

3. Huh Y, Choi I, Song M, Kim S, Hong SD, Joung Y. A comparison of comorbidity and psychological outcomes in children and adolescents with attention-deficit/hyperactivity disorder. Psychiatr Invest 2011; 8: 95-101.

4. Barkley RA, Murphy KR, Fischer M. ADHD in adults: what the science says. New York: Guilford Press; 2008.

5. Sibley M, Pelham WE, Molina SG, Waschbusch DA, Biswas A, MacLean, MG, et al. The delinquency outcomes of boys with ADHD with and without comorbidity. J Abnorm Child Psychol 2011; 39: 21-32.

6. Spencer TJ. ADHD and comorbidity. J Clin Psychiatry 2006; 67: 27-31.

7. Sheese BE, Rothbart MK, Volejer PM, Posner MI. The dopamine receptor D4 gene 7-repeat allele interacts with parenting 
quality to predict effortful control in four year old children. Child Dev Res 2012; 2012: 863242.

8. Smith HJ, Sheikh HI, Dyson MW, Olino TM, Laptook RS, Klein DN. Parenting and child DRD4 genotype interact to predict children's early emerging effortful control. Child Dev 2012; 83: 1932-44.

9. Pennington B. Development of psychopathology: nature and nurture. New York: Guilford Press; 2002.

10. Ellis B, Nigg J. Parenting practices and attention deficit/ hyperactivity disorder: new findings suggest partial specificity of effects. J Am Acad Child Adolesc Psychiatry 2009; 48: 146-54.

11. Hawes DJ, Dadds MR, Frost AD. Parenting practices and prospective levels of hyperactivity/inattention across earlyand middle-childhood. J Psychopathol Behav Assess 2011; 35: 273-82.

12. McLaughlin DP, Harrison CA. Parenting practices of mothers of children with ADHD: the role of maternal and child factors. Child Adolesc Ment Health 2006; 11: 82-8.

13. Miranda-Casas A, Grau-Sevilla D, Marcos-Taberner R, Roselló B. Estilos de disciplina en familias con hijos con trastorno por déficit de atención con hiperactividad: influencia en la evolución del trastorno. Rev Neurol 2007; 44 (Supl 2): S23-5.
14. Kaiser NM, McBurnett K, Piffner LJ. Child ADHD severity and positive and negative parenting as predictors of child social functioning: evaluation of three theoretical models. J Atten Disord 2011; 15: 193-203.

15. Arnold DS, O'Leary SJ, Wolff LS, Acker MM. The parenting scale: a measure of dysfunctional parenting in discipline situations. Psychol Assess 1993; 5: 137-44.

16. Locke LM, Prinz RJ. Measurement of parental discipline and nurturance. Clin Psychol Rev 2002; 22: 895-930.

17. Conners CK. Conners Parent Rating Scale-Revised: long version (CPRS-R:L). Toronto: Multi-Health Systems; 2001.

18. Demaray MK, Elting J, Schaefer K. Assessment of attentiondeficit hyperactivity disorder (ADHD). A comparative evaluation of five, commonly used, published rating scales. Psychol Sch 2003; 40: 341-61.

19. Stormshak EA, Bierman KL, McMahon RJ, Lengua LJ. Parenting practices and child disruptive behavior problems in early elementary school. J Clin Psychol 2000; 29: 17-29.

20. Keown LJ, Woodward LJ. Early parent-child relations and family functioning of preschool boys with pervasive hyperactivity. J Abnorm Child Psychol 2002; 30: 541-53.

\section{Discipline styles and co-morbid disorders of adolescents with attention deficit hyperactivity disorder: a longitudinal study}

Introduction. Problems in cognitive functioning, social and educational development of children with attention deficit hyperactivity disorder (ADHD) continue to be present in adolescence and adulthood. Although the literature shows a significant relationship between the use of dysfunctional discipline methods and severity in the course of ADHD, follow-up studies have been rare.

Aims. To analyze parenting style and ADHD symptomatology assessed in childhood (time 1) to predict the oppositional behavior and cognitive problems in early adolescence (time 2), and to study, depending on the use of dysfunctional parenting style, the course of oppositional behavior and cognitive problems.

Patients and methods. Forty-five children with ADHD-combined presentation were assessed in two different moments: time 1 (ages: 6-13) and time 2 (ages: 8-16).

Results. Oppositionism and cognitive problems in the follow-up were predicted by dysfunctional discipline styles and ADHD severity (assessed in time 1). Oppositional behavior increased between time 1 and time 2 in children with a dysfunctional parenting, whereas a decrease on oppositional symptoms was observed in the functional parenting group (time $\times$ discipline interaction effect).

Conclusions. Dysfunctional parenting practices in childhood predicted cognitive and behavioral problems associated in adolescence. The findings have implications for the planning of interventions.

Key words. ADHD. Cognitive problems. Discipline styles. Longitudinal study. Oppositional behavior. 